\title{
On the Unfolding of a Blowout Bifurcation
}

\author{
Peter Ashwin, Philip J. Aston* and Matthew Nicol ${ }^{\dagger}$
}

April 30, 1997

\begin{abstract}
Suppose a chaotic attractor $A$ in an invariant subspace loses stability on varying a parameter. At the point of loss of stability, the most positive Lyapunov exponent of the natural measure on $A$ crosses zero at what has been called a 'blowout' bifurcation.

We introduce the notion of an essential basin of an attractor with an invariant measure $\mu$. This is the set of points such that the set of measures defined by the sequence of measures $\frac{1}{n} \sum_{k=0}^{n-1} \delta_{f^{k}(x)}$ has an accumulation point in the support of $\mu$. We characterise supercritical and subcritical scenarios according to whether the Lebesgue measure of the essential basin of $A$ is positive or zero.

We study a drift-diffusion model and a model class of piecewise linear mappings of the plane. In the supercritical case, we find examples where a Lyapunov exponent of the branch of attractors may be positive ('hyperchaos') or negative, depending purely on the dynamics far from the invariant subspace. For the mappings we find asymptotically linear scaling of Lyapunov exponents, average distance from the subspace and basin size on varying a parameter. We conjecture that these are general characteristics of blowout bifurcations.
\end{abstract}

\section{Introduction and background}

There has been a lot of recent interest in the global dynamics of systems with attractors in invariant subspaces, with the recognition that the structure of basins of attraction may be very complicated ('riddled basins' [1]). The structure and properties of the basins (including bifurcations) may be understood by examining Lyapunov exponents normal to the invariant subspace. Some relevant work is listed in the bibliography but we mention $[1,4,17,18]$ specifically.

In this paper we investigate blowout bifurcations of chaotic attractors in invariant subspaces and in particular 'on-off' intermittency. The term 'blowout' was coined by Ott \& Sommerer in [18] although the phenomenon was earlier recognised by Pikovsky [19] and Yamada and Fujisaka [25]. There are two types of blowout: subcritical (or hard) bifurcation and supercritical (or soft) bifurcation.

Although we believe that much of what we find will be found in dynamical systems satisfying rather general conditions, our analytical results centre on model piecewise

*Department of Mathematical and Computing Sciences, University of Surrey, Guildford GU2 $5 \mathrm{XH}, \mathrm{UK}$.

${ }^{\dagger}$ Department of Mathematics, UMIST, Manchester M60 1QD, UK. 
linear planar maps and drift-diffusion systems. In particular we investigate the dynamical and ergodic properties of nearby invariant sets in a class of such models. We find that the Lebesgue measure of the basin (subcritical case), the normal Lyapunov exponents and the average distance from the invariant subspace (supercritical case) all scale linearly near the blowout.

We give examples where the supercritical blowout leads to blownout attractors with either positive or negative normal Lyapunov exponents; thus one can get blowout bifurcations that lead to hyperchaos, and ones that do not. This helps to unify the observations of [24] and [13] (who find hyperchaos) with those of Lai [11] (who does not). The presence or absence of hyperchaos is found to depend only on the dynamics far from the invariant subspace.

In order to characterise the sub- and supercritical bifurcations we introduce the notion of an essential basin of attraction. Suppose we have a compact invariant set $A$ for a mapping $f$ of $\mathbf{R}^{n}$. The essential basin of $A, B_{\text {ess }}(A)$, is the set of points which visit each neighbourhood of $A$ not only infinitely often but also infinitely often with a nonvanishing frequency of return. To be more precise, $x \in B_{\text {ess }}(A)$ if for each neighbourhood $U \supset A$,

$$
\limsup _{n \rightarrow \infty} \frac{1}{n} \#\left\{k: f^{k}(x) \in U, 0 \leq k \leq n\right\}>0 .
$$

For the model system we can characterise a blowout as being supercritical if its essential basin has full measure in some neighbourhood of the attractor $A$ at blowout and subcritical if its essential basin has zero measure at blowout. In the general case, this amounts to a conjecture.

The paper is organised as follows: in Section 2 we discuss some generalities of the dynamics at blowout bifurcation and introduce the essential basin of attraction. We discuss the behaviour of the Lyapunov exponents on continuous branches of attractors.

In Section 3 we consider a family of maps of the plane leaving the $x$-axis invariant where we can completely understand the dynamics and scaling behaviours. In fact, we may model the dynamics as a biased random walk on a discrete set of lines or strips. Using properties of the random walk model:

1. We investigate the system at blowout in detail; in particular the properties of the basin (and essential basin) of attraction of $A$.

2. In the supercritical case we find that the average distance of a generic point from the invariant subspace scales linearly with the bifurcation parameter when the system exhibits on-off intermittency. The 'blownout' attractors may be viewed as belonging to a continuous branch of attractors bifurcating from the invariant subspace attractor.

3. We find scenarios where the blowout leads to hyperchaos and scenarios where it does not.

4. We recover results of $[10,17]$ on the distribution of laminar phases in on-off intermittency and in the subcritical case (before blowout) we show that the 
measure of the basin of attraction also scales linearly with the bifurcation parameter.

Finally, in Section 4 we address further problems and generalisations of this approach.

\section{Essential basins and branching of chaotic at- tractors}

Ott and Sommerer [18] first noted that a system near a blowout bifurcation (characterised by a normal Lyapunov exponent passing through zero) may exhibit on-off intermittency, riddled basins and a variety of related behaviours. They conjectured the existence of two scenarios:

1. A hysteretic (subcritical) scenario where riddled basins before the blowout give rise to a 'hard' loss of stability (Pikovsky [19]). In other words, after blowout, almost all points near the invariant subspace eventually move away, never to return.

2. A non-hysteretic (supercritical) scenario giving a 'soft' loss of stability to an 'on-off intermittent' attractor [21]. The characteristic feature of this scenario is that trajectories return close to the invariant subspace and remain nearby for long periods of time.

In [4] it was shown that before blowout bifurcation both scenarios display 'locally riddled basins': in any neighbourhood of the attractor there is a positive measure set of points whose trajectories iterate to a uniform distance from the attractor. These trajectories are either re-injected to the neighbourhood or are lost to the basin of another attractor. For the model in Section 3 we show that we can distinguish between sub- and supercritical blowout depending on whether the essential basin of the attractor in the invariant subspace has positive measure or not at the point of blowout.

Suppose $f: M \rightarrow M$ is a locally invertible smooth map on some compact open region $M \subset \mathbf{R}^{n}$ and $\ell_{M}$ is Lebesgue measure on $M$. For a compact set $B \subset M$ define

$$
\mathcal{M}(B)=\{\mu: \mu \text { is an } f \text {-invariant measure with } \operatorname{supp}(\mu) \subset B \text { and } \mu(B)=1\}
$$

and equip this with the $C^{*}$ topology. Let $\omega(x)$ denote the set of accumulation points of $\left\{f^{n}(x)\right\}$ in $M$ and let $\delta_{x}$ denote the point measure at the point $x \in M$. We let $\Omega(x)$ be the set of accumulation points of the sequence of measures

$$
\frac{1}{n} \sum_{k=0}^{n-1} \delta_{f^{k}(x)}
$$

Any accumulation points will lie in $\mathcal{M}(M)$. We define the essential $\omega$-limit set of $x$ by

$$
\omega_{e s s}(x)=\overline{\cup_{\mu \in \Omega(x)} \operatorname{supp}(\mu)} .
$$

Note that compactness of $M$ implies that $\mathcal{M}(M)$ is also compact and therefore $\omega(x)$ and $\omega_{\text {ess }}(x)$ are non-empty; trivially $\omega(x) \supset \omega_{\text {ess }}(x)$. The next result follows directly from the definition. 
Lemma 1 The essential $\omega$-limit set of an initial point $x$ can be alternatively characterised as the set of $y$ such that, for all $\epsilon>0$, the limit

$$
\limsup _{n \rightarrow \infty} \frac{1}{n} \#\left\{0 \leq i<n:\left|f^{i}(x)-y\right|<\epsilon\right\}>0 .
$$

In other words, the $\omega_{e s s}$ is the set of all limit points that contribute to averages along the trajectory of $x$. By the above $\omega(x) \supset \omega_{\text {ess }}(x)$ but typically they can differ (for example, see the discussion of Sigmund [23]). We can use this to define a generalisation of the usual notion of a basin of attraction. Suppose $A \subset M$; recall that the (topological) basin of attraction is defined as

$$
B(A)=\{x \in M: \omega(x) \subset A\} .
$$

An attractor for a mapping $f$ is a compact, invariant set $A$ such that $\ell_{M}(B(A))>0$. By a natural ergodic measure $\mu$ for an attractor $A$ we mean a measure such that $\lim _{n \rightarrow \infty} \frac{1}{n} \sum_{i=0}^{n} \phi\left(f^{i}(x)\right)=\int_{A} \phi d \mu$ for all continuous functions $\phi$ and Lebesgue almost every $x$ in $B(A)$.

Definition 2 We define the essential basin as

$$
B_{e s s}(A)=\left\{x \in M: \omega_{e s s}(x) \subset A\right\} .
$$

Note that

$$
B(A) \subset B_{e s s}(A) .
$$

The essential basin of an attractor can be much bigger than the basin. For example, consider an attracting homoclinic cycle; the basin of attraction of the equilibrium is just the cycle while the essential basin will be a neighbourhood of the cycle.

\subsection{Branching of chaotic attractors from invariant subspaces}

We introduce the notion of a branch of attractors, characterised by continuity of the family of natural invariant measures supported on them. For periodic orbits or fixed points this corresponds to the usual idea of a branch. Unfortunately these cannot be expected to exist for non-uniformly hyperbolic attractors, but they can exist for idealised families, especially such as those arising from skew product or chaotically forced systems and we suspect that they may exist as continuous families on sets in parameter space with large measure.

Definition 3 A branch of attractors $\left(A_{\epsilon}, m_{\epsilon}\right)$ is a family of $f_{\epsilon}$-invariant sets $A_{\epsilon}$ supporting ergodic natural measures $m_{\epsilon}$ such that $m_{\epsilon}$ is a continuous map from $\mathbf{R}$ to $\mathcal{M}(M)$.

Suppose now that $f$ leaves a smoothly embedded codimension one submanifold $N$ invariant, such that $\left.f\right|_{N}$ has a chaotic attractor $A$ supporting a natural measure $\mu_{A}$ (we restrict to codimension one invariant subspaces for simplicity). As in Ashwin et al. [4] we define the normal Lyapunov spectrum for the attractor $A$ to be the set of normal Lyapunov exponents for all ergodic invariant measures supported on $A$. We write $\lambda_{\perp}(\mu)$ to be the most positive normal Lyapunov exponent of an ergodic 
invariant measure $\mu$. As discussed in [4], if there is non-trivial (chaotic) dynamics on $A$ then generically there are ergodic invariant measures $\mu_{\min }$ and $\mu_{\max }$ such that

$$
\lambda_{\perp}\left(\mu_{\min }\right)<\lambda_{\perp}\left(\mu_{A}\right)<\lambda_{\perp}\left(\mu_{\max }\right)
$$

where the inequalities are strict and $\mu_{A}$ is the natural measure for $\left.f\right|_{N}$ (supported on A).

We consider smooth one-parameter families of maps $f_{\epsilon}: M \rightarrow M$ such that $f_{0}$ is at a blowout bifurcation:

Definition 4 A branch of attractors $\left(A_{\epsilon}, m_{\epsilon}\right)$ for $\left.f_{\epsilon}\right|_{N}$ undergoes a blowout bifurcation at $\epsilon=0$ if $\ell_{M}\left(B\left(A_{\epsilon}\right)\right)>0$ for $\epsilon<0$ and $\ell_{M}\left(B\left(A_{\epsilon}\right)\right)=0$ for $\epsilon>0$.

From [4, Theorems 2.19, 2.20] and the definition of a blowout bifurcation it follows that the normal Lyapunov exponent $\lambda_{\perp}\left(m_{0}\right)$ is zero at $\epsilon=0$ and is continuous in $\epsilon$. Typically a blowout bifurcation occurs where $\lambda_{\perp}\left(m_{\epsilon}\right)<0$ for $\epsilon<0$ and $\lambda_{\perp}\left(m_{\epsilon}\right)>0$ for $\epsilon>0$.

If the only branch of attractors emanating from a blowout bifurcation is contained in the invariant subspace, we say it is subcritical. Otherwise we say the blowout is supercritical. The branch of attractors for $\epsilon>0$ in the supercritical case exhibits on-off intermittency $[18,21]$ and they are stuck on [3] to $N$.

We discuss basic properties of branches near blowout bifurcations. Suppose now that $f_{\epsilon}$ is a smooth one-parameter family of maps that is a skew product over a fixed map $\left.f_{0}\right|_{N}$ on the invariant subspace, i.e. we assume we can write the map in the form

$$
\begin{aligned}
& x_{n+1}=g\left(x_{n}\right) \\
& y_{n+1}=h_{\epsilon}\left(x_{n}, y_{n}\right)
\end{aligned}
$$

with $x_{n} \in N$ and $y_{n} \in \mathbf{R}$ where $g$ has an attractor $A_{0}$ in the invariant subspace $N$ (note that $h_{\epsilon}(x, 0)=0$ ). The skew product structure means that one of the Lyapunov exponents always corresponds to the rate of growth of tangent vectors in the normal direction. In this case the normal Lyapunov exponent measures the rate of growth of the normal derivative $D f_{\epsilon}^{\perp}=\frac{\partial h_{\epsilon}}{\partial y}$. The normal Lyapunov spectrum of $A$ is continuous in $\epsilon$ in the case of codimension one invariant subspaces [4].

Theorem 5 Suppose $\left(A_{0}, m_{0}\right)$ undergoes a blowout bifurcation at $\epsilon=0$. Any branch $\left(\tilde{A}_{\epsilon}, \tilde{m}_{\epsilon}\right)$ of attractors with $\tilde{m}_{0}=m_{0}$ must also have a normal Lyapunov exponent that limits to zero at $\epsilon=0$.

Proof The Lyapunov exponent of $\tilde{A}_{\epsilon}$ in the normal direction is given by the expression

$$
\int \log ^{+}\left(\left|D f_{\epsilon}^{\perp}(x)\right|\right) d \tilde{m}_{\epsilon}
$$

(as a result of the ergodicity of $f_{\epsilon}$ with respect to $\tilde{m}_{\epsilon}$ ). When evaluated at $\epsilon=0$ this gives zero since $\tilde{A}_{0}=A_{0}$. By continuity of $\log ^{+}\left(\left|D f_{\epsilon}^{\perp}(x)\right|\right)$ in $\epsilon$ and the continuity of $d \tilde{m}_{\epsilon}$ in the $\mathcal{C}^{*}$ topology with $\epsilon$ we see that the normal Lyapunov exponent of $\tilde{A}_{\epsilon}$ must limit to zero as $\epsilon$ tends to zero.

QED 


\subsection{Unfolding the blowout; criticality}

Suppose that $f_{0}$ has an invariant set $A_{0}$ at the blowout bifurcation i.e. $\lambda_{\perp}\left(m_{0}\right)=0$, and suppose that $f_{\epsilon}$ is a parametrised family of maps with an associated branch of attractors $\left(A_{\epsilon}, m_{\epsilon}\right)$. We can generically assume that $f_{\epsilon}$ unfolds the blowout, i.e. $\lambda_{\perp}\left(m_{\epsilon}\right)<0$ for $\epsilon<0$ and $\lambda_{\perp}\left(m_{\epsilon}\right)>0$ for $\epsilon>0$.

We conjecture there are links between the essential basin of attraction at the point of blowout bifurcation and the criticality of the blowout.

Subcritical case. If $B_{\text {ess }}(A)$ has zero $\ell_{M}$-measure we conjecture that generically the unfolding gives a subcritical scenario. In this case there can be no attracting invariant sets intersecting a neighbourhood $U_{A}$ of $A$ and therefore no branches of nearby attractors for $\epsilon>0$. The fact that the essential basin of $A$ has no measure in $U_{A}$ does not mean that almost all points leave $U_{A}$ never to return; however, we believe that this is typically the case.

Supercritical case If $B_{\text {ess }}(A)$ has positive $\ell_{M}$-measure in some neighbourhood $U_{A}$ of $A$ we conjecture that the blowout is generically supercritical, i.e. we conjecture there is typically a continuous branch of attractors $\left(\tilde{A}_{\epsilon}, \tilde{m}_{\epsilon}\right)$ bifurcating from the invariant subspace for $\epsilon>0$.

\subsection{Drift-diffusion model}

We now verify that the conjecture holds when $M$ has dimension two, the blowout is supercritical and can be modelled by a one-dimensional drift-diffusion model $[7,10$, $16,17,20]$. Assume that the dynamics take place on a unit square $[0,1] \times[0,1]$ with the invariant subspace being the unit interval $[0,1]$ on the $x$-axis and that trajectories going into $y>1$ are reinjected back at $y=1$. We make a change of coordinates and let $z=-\log y$; in these coordinates the 'reinjection' takes place at $z=0$ and we assume that the system is near blowout (so that the drift is close to zero). The drift-diffusion model has two parameters $\lambda$ and $D$ and

$$
\lambda=\langle\delta z\rangle \text { and } D=\frac{1}{2}\left\langle(\delta z-\langle\delta z\rangle)^{2}\right\rangle
$$

where $\langle\cdot\rangle$ indicates expected value and $\delta z=z_{n+1}-z_{n}$. A probability distribution $P(z, t)$ evolves according to the equation $[17]$

$$
\frac{\partial P}{\partial t}=\lambda \frac{\partial P}{\partial z}+D \frac{\partial^{2} P}{\partial z^{2}}
$$

As in [17], $\lambda$ is the Lyapunov exponent while $D$ can be interpreted as the scaling of the standard deviation of finite-time Lyapunov exponents.

The reinjection at $z=0$ is modelled by a zero probability flux at $z=0$; this together with the normalisation condition gives the boundary conditions

$$
\left.\left(\lambda P+D \frac{d P}{d z}\right)\right|_{z=0}=0, \quad \int_{0}^{\infty} P(z, t) d z=1 .
$$


The drift-diffusion equation has a steady state solution satisfying these boundary conditions given by $P(z)=\frac{\lambda}{D} e^{-\frac{\lambda}{D} z}$ which is normalisable in $z \in(0, \infty)$ precisely if $\lambda>0$; all initial probability distributions will evolve towards this one. In terms of the variable $y$ we get a density

$$
\hat{P}(y)=\frac{\lambda}{D} y^{\frac{\lambda}{D}}\left|\frac{d z}{d y}\right|=\frac{\lambda}{D} y^{-1+\frac{\lambda}{D}} .
$$

Translating this back to the original problem gives a continuous branch of attractors with these normal invariant probability densities for $\lambda>0$.

In the limit of $\lambda=0$ (at the point of blowout bifurcation) this model gives a nonnormalisable invariant measure with density proportional to $y^{-1}$ and corresponds to having an essential basin of attraction for the invariant subspace with full Lebesgue measure. In contrast, the measure of the (topological) basin of the attractor is zero, because almost all trajectories display large deviations away from the invariant subspace after arbitrarily long times. Moreover, the scalings of average distance of the invariant measures for $\lambda>0$ can be computed to scale linearly with the Lyapunov exponent which may be taken as a generic unfolding parameter.

\section{A model piecewise linear map of the plane}

We consider in some detail a modification of a family of mappings proposed by [7, $10,16,17]$. This is a three parameter family of mappings of $[0,1] \times \mathbf{R}$ to itself defined by

$$
f(x, y)=(g(x), h(x, y))
$$

where

$$
g(x)= \begin{cases}\frac{x}{\alpha} & \text { for } 0 \leq x \leq \alpha \\ \frac{x-\alpha}{1-\alpha} & \text { for } \alpha<x \leq 1\end{cases}
$$

and

$$
h(x, y)= \begin{cases}\gamma y & \text { if } y<1 \text { and } 0 \leq x \leq \alpha \\ \gamma^{-1} y & \text { if } y<1 \text { and } \alpha<x \leq 1 \\ 1+\beta(1-y) & \text { if } y \geq 1\end{cases}
$$

We assume a symmetry $y \mapsto-y$ to define the map on the lower half plane. The parameters are $\alpha \in(0,1), \gamma>0$ and $\beta \in \mathbf{R}$.

We note that

$$
A=[0,1] \times\{0\}
$$

is an invariant subspace for the map (3.2) whose basin of attraction $B(A)$ may or may not have positive measure.

Without loss of generality we can assume that $\gamma>1$ (if $\gamma=1$ the dynamics near the $x$-axis are trivial). This mapping can be used to investigate both supercritical and subcritical blowout bifurcations of $A$. When we vary $\alpha$ through $\frac{1}{2}$ it exhibits a supercritical blowout for small $\beta>0$ and a subcritical blowout for small $\beta<0$. 
Properties of the base transformation, $g$. The Markov map $g$ can be conjugated on a set of full Lebesgue measure to a semi-shift on two symbols with state space $\{0,1\}^{\mathbf{N}}$ defined in the usual way by mapping $x$ to the sequence $\left\{\omega_{i}\right\}_{i \in \mathbf{N}}$ where $\omega_{i}=0$ if $f^{i}(x)$ lies in the interval $[0, \alpha)$ and $\omega_{i}=1$ if $f^{i}(x)$ lies in the interval $(\alpha, 1]$.

For every $p \in[0,1]$ there is a closed invariant set $C_{p} \subset[0,1]$ supporting a measure $m_{p}$ corresponding to symbol sequences where the 0 appears with average frequency $p$. The normal Lyapunov exponent of the measure $m_{p}$ can be easily computed to give

$$
\lambda_{\perp}\left(m_{p}\right)=(2 p-1) \log |\gamma|
$$

and so for any $\alpha \in(0,1)$ the map defined by this choice of $\alpha$ has uncountably many invariant measures $\left\{m_{p}\right\}$ with both positive and negative normal Lyapunov exponents.

For a given $0<\alpha<1$ the measure $m_{\alpha}$ is equivalent to Lebesgue measure on $[0,1]$, and so $m_{\alpha}$ is a natural ergodic invariant measure for $g$; the normal Lyapunov exponent is determined as above. If we define $\epsilon$ by

$$
\alpha=\left(\frac{1+\epsilon}{2}\right)
$$

then we can vary $\epsilon \log \gamma$, the normal Lyapunov exponent.

There is a countable infinity of periodic measures and other aperiodic invariant measures associated with (for example) adding machines. In short, the ergodic structure of the mapping $g$ is complicated but well understood.

\subsection{Supercritical blowout; $\beta>0$}

Invariant lines. Assume that $\beta>0$. For each $N=1,2, \ldots$, if there is a solution $y_{N}$ of

$$
y_{N}=1+\beta\left(1-\gamma^{N} y_{N}\right)
$$

such that $0<\gamma^{k} y_{N}<1$ for $k=1, \cdots, N-1$ and $\gamma^{N} y_{N}>1$, then there is a set of lines

$$
L_{N}=\left\{\left(x, \gamma^{N-n} y_{N}\right): x \in[0,1], n=0,1, \ldots\right\}
$$

that is invariant under $f$. Note that the inequality $\gamma^{N-1} y_{N}<1$ implies that we can find such a $y_{N}$ if $\beta$ satisfies

$$
\frac{\gamma^{N-1}-1}{\gamma^{N}-\gamma^{N-1}}<\beta
$$

In particular we can have a number of simultaneously existing invariant line families corresponding to differing values of $N$.

Note that the existence of such a family of lines is independent of the parameter $\alpha$ (although the dynamics on and near them is not). On such an invariant set of lines we can completely understand the dynamics by observing that the $y$ dynamics is a random walk on non-negative integers. If we assume that

$$
0<\beta<\beta^{*} \equiv \frac{1}{\gamma}
$$

then there only exists one set of invariant lines, namely $L_{1}$. 
The random walk on $L_{1}$. The map $f$ restricted to $L_{1}$ is conjugate to a map

$$
T:[0,1] \times L_{1} \rightarrow[0,1] \times L_{1}
$$

defined by

$$
T(x, n)=(g(x), m(x, n))
$$

where

$$
m(x, n)= \begin{cases}n-1 & \text { if } x \leq \alpha \text { and } n \geq 1 \\ n+1 & \text { if } x>\alpha \text { and } n \geq 1 \\ 1 & \text { if } n=0\end{cases}
$$

The second component of $T$ is a Markov process on non-negative integers with the non-zero transition probabilities given by

$$
\begin{array}{ll}
p_{i, i+1}=\frac{1-\epsilon}{2} & \text { for } i \geq 1 \\
p_{i, i-1}=\frac{1+\epsilon}{2} & \text { for } i \geq 1 \\
p_{0,1}=1 &
\end{array}
$$

Steady state distributions and ergodic measures. The steady state probability distribution is found by solving the recurrence relation

$$
P_{i}=\frac{1-\epsilon}{2} P_{i-1}+\frac{1+\epsilon}{2} P_{i+1}
$$

with initial condition $P_{2}=\frac{1-\epsilon}{1+\epsilon} P_{1}$ (note that $P_{0}=\frac{1+\epsilon}{2} P_{1}$ ). The solution for this recurrence relation $(n \geq 1)$ is $P_{n}=A \Lambda^{n}+B$ where

$$
\Lambda=\frac{1-\epsilon}{1+\epsilon} .
$$

If and only if $\epsilon>0$, we can normalise the invariant measure $\sum_{n=0}^{\infty} P_{n}=1$ giving $B=0$ and

$$
A(\epsilon)=\frac{2 \epsilon}{1-\epsilon^{2}}
$$

and so

$$
P_{n}=\frac{2 \epsilon}{1-\epsilon^{2}} \Lambda^{n} \quad(n \geq 1), \quad P_{0}=\frac{\epsilon}{1+\epsilon} .
$$

There are dense orbits in $L_{1}$ for any $\epsilon$.

For $\beta>0$ we obtain a supercritical scenario where an attractor blows out at $\alpha=\frac{1}{2}$ to a family of on-off intermittent attractors. For small $0<\beta<\beta^{*}$ we get a blowout to an attractor supported on $L_{1}$ with one positive Lyapunov exponent; for larger $\beta>0$ we can get blowout to an attractor with two positive Lyapunov exponents (hyperchaos) supported on a two dimensional set. We deal with these cases separately. Note that in both cases Theorem 5 implies that the normal Lyapunov exponent must go to zero at the point of bifurcation. 


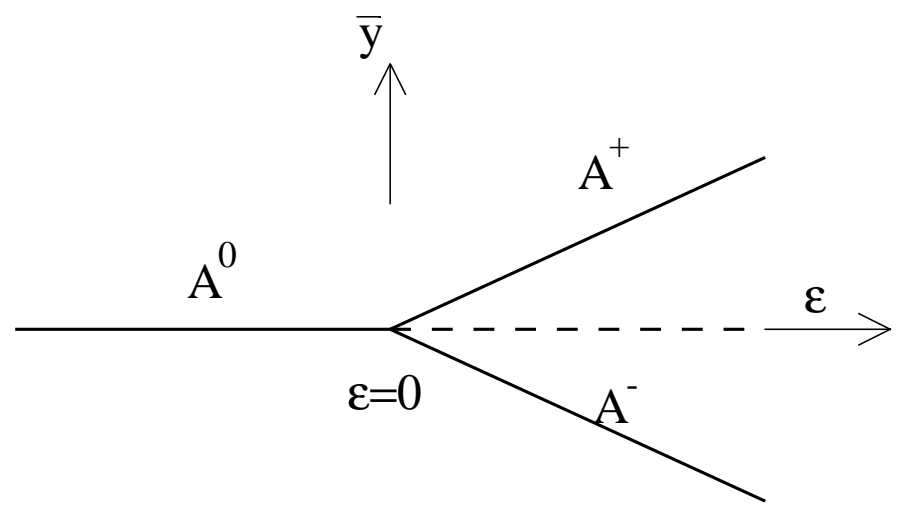

Figure 1: A schematic representation of a supercritical blowout bifurcation, plotting $\bar{y}$ (the average value of the normal coordinate) against the parameter $\epsilon$. Note the linear scaling with $\epsilon>0 . A^{+}$and $A^{-}$are symmetrically related 'blown out' attractors.

\subsection{Blowout to attractor with one positive Lyapunov expo- nent}

We assume that $0<\beta<\beta^{*}$; although there is no Lyapunov exponent defined in the vertical direction for the dynamics on $L_{1}$ (this is a one-dimensional set), we can compute one for the dynamics embedded in the plane. Note that the dynamics in the $y$-direction consists of simple scalings dependent on the $y$-itinerary of a given point. In fact we can write the $y$-Lyapunov exponent for $L_{1}$ as

$$
\lambda_{\perp}\left(L_{1}\right)=\log (\beta \gamma) P_{0}=\log (\beta \gamma) \frac{\epsilon}{1+\epsilon} .
$$

This can be seen if we examine the returns of an orbit to 0 in $L_{1}$; the total expansion or contraction will be the product $\gamma \beta$; weighting this by the frequency of visits to 0 gives the result. It is clear that $\lambda_{\perp}\left(L_{1}\right)$ is negative for $\beta<\frac{1}{\gamma}$, and to first order it scales linearly with $\epsilon$. An example of an attracting $L_{1}$ is shown in Figure 2(a) after transients have died away. For larger positive values of $\beta$ it is possible to get more than one set of lines simultaneously stable. In this case we note that the blowout bifurcation creates multiple branches of attractors. Whether this is possible for typical systems remains to be seen.

Schematically, we represent the blowout bifurcation to riddled basins on varying $\epsilon$ by Figure 1. This is analogous to a pitchfork bifurcation of equilibria, and the scaling of the Lyapunov exponents is linear with $\epsilon$. There is a crucial difference that the average distance from the invariant subspace scales linearly rather than as a square-root (see Section 3.4).

\subsection{Blowout to attractor with two positive Lyapunov expo- nents (hyperchaos)}

For motivation, we first consider the map with only expanding components given by

$$
y_{n+1}=\tilde{h}\left(y_{n}\right)
$$


(and trivial action on the $x$ variable) where

$$
\tilde{h}(y)= \begin{cases}\gamma y & \text { if } y<1 \\ 1+\beta(1-y) & \text { if } y \geq 1\end{cases}
$$

We define the strips

$$
I_{n}=[0,1] \times\left(\gamma^{-n-1}, \gamma^{-n}\right), \quad n=-1,0,1,2,3, \ldots
$$

The height of the strip $I_{n}$ is $h_{n}=\gamma^{-n-1}(\gamma-1)$; clearly $h_{n} \rightarrow 0$ as $n \rightarrow \infty$.

If we set $\beta=\frac{\gamma+1}{\gamma^{2}}$ and $1<\gamma<\frac{1+\sqrt{5}}{2}$ (so that $1<\beta$ ) then the action of the map is such that

$$
\tilde{h}\left(I_{1}\right)=I_{0}, \quad \tilde{h}\left(I_{0}\right)=I_{-1}, \quad \tilde{h}\left(I_{-1}\right)=I_{1} \cup I_{0}
$$

if we restrict to the full measure subset of $I_{n}$ that never iterates to the discontinuity. There is an invariant set with full measure in the union of the strips $I_{n}, n=-1,0,1$. Note that the map $\tilde{h}$ is everywhere expanding and we show that it can also be conjugated to a random walk model described in terms of the measure $d_{n}$ on the strip $I_{n}$.

By considering the Frobenius-Perron equation for this map, it can be shown that the natural probability density is constant on each of the three strips $I_{n}, n=-1,0,1$. If the natural measure on each strip is given by $\tilde{d}_{n}$ then it can be shown that

$$
\tilde{d}_{-1}=\frac{\gamma+1}{2 \gamma+3}, \quad \tilde{d}_{0}=\frac{\gamma+1}{2 \gamma+3}, \quad \tilde{d}_{1}=\frac{1}{2 \gamma+3} .
$$

The Lyapunov exponent for this attractor can be evaluated and is given by

$$
\begin{aligned}
\tilde{\lambda} & =\left(\tilde{d}_{1}+\tilde{d}_{0}\right) \log \gamma+\tilde{d}_{-1} \log \beta \\
& =\left(\frac{\gamma+2}{2 \gamma+3}\right) \log \gamma+\left(\frac{\gamma+1}{2 \gamma+3}\right) \log \beta
\end{aligned}
$$

Clearly $\tilde{\lambda}>0$ provided that $\beta>1$ as expected.

We now reintroduce the contracting part of the normal dynamics and consider again the model where we have both expansion and contraction by a factor of $\gamma$ or $\gamma^{-1}$ respectively. In this case, the iterates can escape from the strip $I_{1}$ and iterate towards the invariant subspace. Note however that as long as $y_{n}<1$, the iterates will move up and down on a particular set of invariant lines, since $y_{n}$ is multiplied by either $\gamma$ or $\gamma^{-1}$ at each iteration. Only when $y_{n}$ exceeds 1 for some $n$ will the set of lines be changed on which the iterates move.

The map $f$ maps strips into strips and so the density on each strip will be constant. The random walk model for the invariant lines can therefore be applied in this example in terms of the total measure $d_{n}$ on the strip $I_{n}$ giving

$$
d_{n}=\left(\frac{1-\epsilon}{2}\right) d_{n-1}+\left(\frac{1+\epsilon}{2}\right) d_{n+1}, \quad n=2,3, \ldots
$$

To derive the initial condition we consider the measure on the three strips $I_{1}, I_{0}$ and $I_{-1}$; this leads to the equations

$$
d_{1}=\frac{h_{1}}{h_{0}+h_{1}} d_{-1}+\left(\frac{1-\epsilon}{2}\right) d_{0}+\left(\frac{1+\epsilon}{2}\right) d_{2}
$$




$$
\begin{aligned}
d_{0} & =\frac{h_{0}}{h_{0}+h_{1}} d_{-1}+\left(\frac{1+\epsilon}{2}\right) d_{1} \\
d_{-1} & =\left(\frac{1+\epsilon}{2}\right) d_{0} .
\end{aligned}
$$

Simplifying equation (3.5) using (3.6) and (3.7) gives the initial condition

$$
d_{2}=\Lambda d_{1}
$$

where $\Lambda=\frac{1-\epsilon}{1+\epsilon}$ as before. The solution of the recurrence relation (3.4) together with the initial condition (3.8) is then

$$
d_{n}=c \Lambda^{n}, \quad n=1,2, \ldots
$$

and from equations (3.6) and (3.7) we obtain

$$
\begin{aligned}
d_{0} & =c \frac{(1-\epsilon)(\gamma+1)}{2+\gamma(1-\epsilon)} \\
d_{-1} & =c \frac{\left(1-\epsilon^{2}\right)(\gamma+1)}{2(2+\gamma(1-\epsilon))} .
\end{aligned}
$$

The constant $c$ can be found from the normalisation condition $\sum_{n=-1}^{\infty} d_{n}=1$ which gives

$$
c=\frac{2 \epsilon(2+\gamma(1-\epsilon))}{\left(1-\epsilon^{2}\right)(2+\epsilon+\gamma(1+\epsilon))}=2 \epsilon+O\left(\epsilon^{2}\right) .
$$

The normal Lyapunov exponent for this attractor is therefore given by

$$
\begin{aligned}
\lambda_{\perp} & =\sum_{n=0}^{\infty} d_{n}\left[\left(\frac{1+\epsilon}{2}\right) \log \gamma+\left(\frac{1-\epsilon}{2}\right) \log \left(\gamma^{-1}\right)\right]+d_{-1} \log \beta \\
& =\frac{\epsilon}{2+\epsilon+\gamma(1+\epsilon)}[(\gamma+2) \log \gamma+(\gamma+1) \log \beta] \\
& =\epsilon\left(\log \gamma+\left(\frac{\gamma+1}{\gamma+2}\right) \log \beta\right)+O\left(\epsilon^{2}\right) .
\end{aligned}
$$

The coefficient of $\epsilon$ in $\lambda_{\perp}$ is clearly positive whenever $\beta>1$. Thus, for small $\epsilon$ and $1<\gamma<1.618$, the blown out attractor has two positive Lyapunov exponents and so is hyperchaotic. An example of such a hyperchaotic attractor is shown in Fig. 2(b). We note that, as in the previous example, the normal Lyapunov exponent goes to zero as the blowout bifurcation is approached.

The natural density on the strip $I_{n}$ at $y \sim Y_{n}=\gamma^{-n}$ is given by

$$
p_{n}=\frac{d_{n}}{h_{n}}=\frac{c \gamma}{\gamma-1} Y_{n}^{\kappa-1}
$$

where

$$
\kappa=-\frac{\log \Lambda}{\log \gamma}=\frac{2}{\log \gamma} \epsilon+O\left(\epsilon^{3}\right) .
$$

Pikovsky and Grassberger [20] study a model synchronisation problem and obtain a normal density function proportional to $y^{\kappa-1}$ with $\kappa=0$ at the blowout bifurcation. This is also consistent with the normal density obtained from the drift-diffusion model in section 2.3. The normal density in this example is a piecewise constant approximation to this function. 


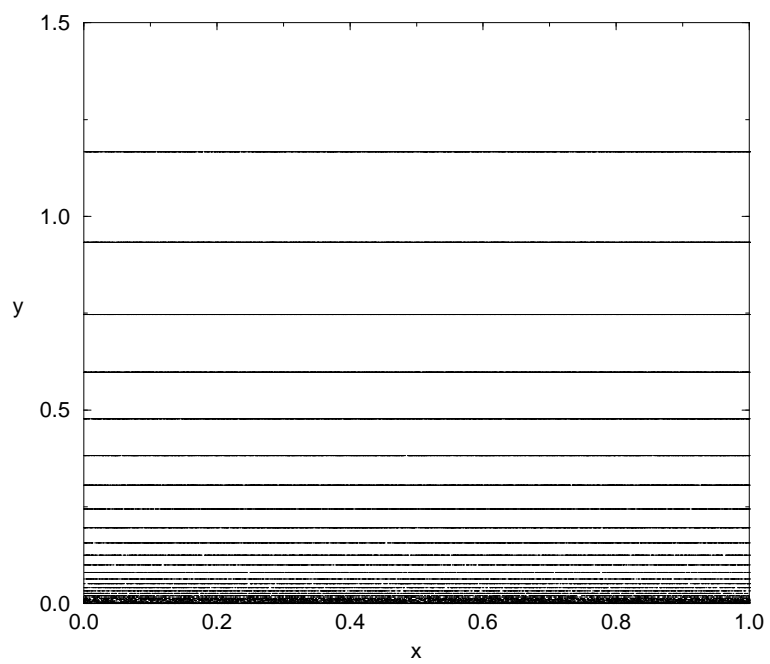

(a)

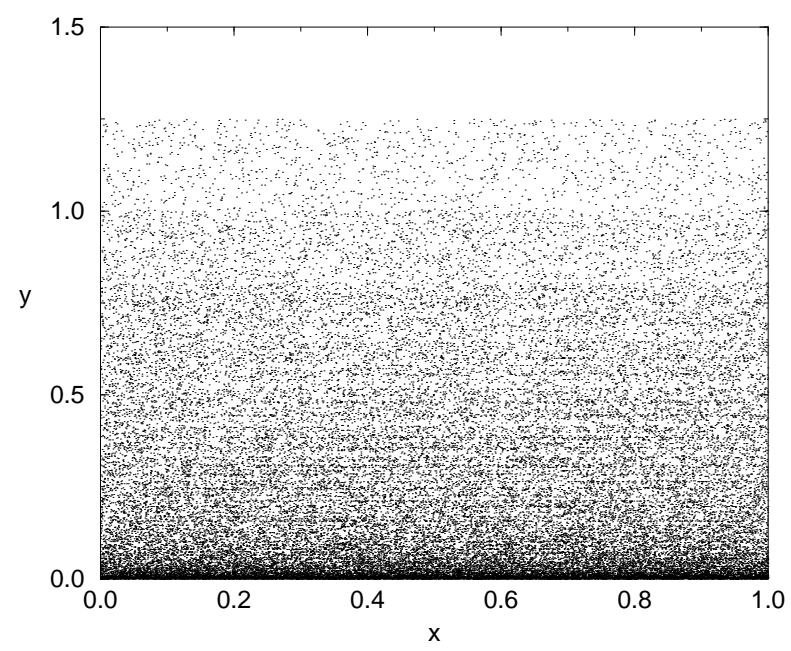

(b)

Figure 2: Attractors for supercritical blowouts at $\gamma=1.25, \epsilon=0.04$ and (a) $\beta=0.4$; an attractor is supported on a countable set of lines and has one positive and one negative Lyapunov exponent. (b) $\beta=1.44$; a hyperchaotic attractor is supported on a rectangle $[0,1] \times[0, \gamma]$. There is evidence of the predicted 'steps' in the natural invariant density. 


\subsection{Scaling near supercritical blowout}

Basin size before blowout We demonstrate that there is a neighbourhood of $A$ containing a full measure set of initial points that converge to $A$ before the blowout.

Theorem 6 For $0<\beta<1 /(\gamma-1)$ and $0<\alpha<\frac{1}{2}$ in (3.2) the basin of $A$ has full measure in $[0,1] \times[0, \gamma]$.

Proof Note that $\beta<1 /(\gamma-1)$ implies that all images of $y \in[0, \gamma]$ under the map are within $[0, \gamma]$. If $\alpha<\frac{1}{2}$ a full measure set (with respect to Lebesgue measure) of $x$ in $[0,1]$ are such that $f^{n}(x, y) \rightarrow A$ for every $y \in[0,1]$. This is because for almost every $x \in[0,1]$ the frequency of times that $f^{n}(x, y)$ moves upwards in the $y$ direction tends to $\alpha$. The frequency of times that the map in the $y$ direction can take the form $1-\beta(1-y)$ is at most $\alpha$ (since we must have at least one upward step for each downward step of the form $1-\beta(1-y)$ ) and hence asymptotically there is a net drift towards $y=0$ at an exponential rate of at least $\gamma^{\epsilon}$ in $n$ i.e. the $y$ coordinate of $f^{n}(x, y)$ tends to 0 faster than $\gamma^{n \epsilon}$ asymptotically in $n$ for almost every $x \in[0,1]$.

QED

Theorem 7 For $0<\beta<1 /(\gamma-1)$ and $\alpha=\frac{1}{2}$ the essential basin of attraction of $A$ has full measure in $[0,1] \times[0, \gamma]$ whereas the basin of attraction of $A$ has zero measure.

Proof For $\alpha=\frac{1}{2}$, close to the invariant subspace there is an unbiased random walk in the logarithm of the $y$ variable. Since this is almost surely recurrent, almost all initial $y \in[0, \gamma]$ do not have $\omega$-limit sets contained in $A$ and therefore the basin of $A$ has zero measure.

The only invariant measure supported in $[0,1] \times(0, \gamma]$ that projects to Lebesgue measure on the $x$ direction is given by a Markov model as above, and this is not normalisable and therefore not in $\mathcal{M}([0,1] \times[0, \gamma])$. As noted in Section 2 the essential $\omega$-limit set of an initial point must be contained in $\mathcal{M}([0,1] \times[0, \gamma])$. Thus for a set of full Lebesgue measure in $[0,1] \times[0, \gamma]$ the natural invariant measure supported on $A$ is the essential $\omega$-limit set of these points and therefore the essential basin of $A$ has full measure.

QED

Mean distance from invariant subspace after blowout. We calculate the mean distance from the invariant subspace $y=0$ for a typical orbit $(x, y)$ under iteration of $f$ for the dynamics on an attracting $L_{1}$. Note that the lines are located at $y=K \gamma^{-n}$ with $K$ constant. The mean distance $\bar{y}$ for this attractor is given by

$$
\begin{aligned}
\bar{y} & =\sum_{n=0}^{\infty} K \gamma^{-n} P_{n} \\
& =K \frac{\epsilon}{1+\epsilon}+K \frac{2 \epsilon}{1-\epsilon^{2}} \sum_{i=1}^{\infty}\left(\frac{\Lambda}{\gamma}\right)^{n} \\
& =\epsilon K\left(\frac{2 \gamma}{\gamma-1}+1\right)+O\left(\epsilon^{2}\right)
\end{aligned}
$$

as $\epsilon \rightarrow 0^{+}$. Thus, to leading order, the average distance of the orbit from the invariant subspace is linear in $\epsilon$. A similar result is obtained for the case of hyperchaotic attractors. 
Distribution of laminar phases for on-off intermittency. To further validate the model, we recover the previous results of [10] on the distribution of laminar phases for on-off intermittency and additionally their scaling with $\epsilon$. A laminar phase is a portion of an orbit that stays close to the invariant subspace and then moves away in a "burst" to a certain threshold level $\tau$. To determine the statistics governing this behaviour in our system we consider an asymmetric random walk $\{X(k)\}_{k \geq 0}$ starting at a state $n$ (i.e. $X(0)=n$ ), which hits the threshold state $\tau=k<n$ for the first time after $m$ steps.

In other words $m$ is the least positive integer such that $X(m)=k$. We suppose that the probability of moving to a lower state is given by $\frac{(1-\epsilon)}{2}$ and the probability of moving to a higher state is $\frac{(1+\epsilon)}{2}$. Let

$$
S_{m}(k)=\{\text { probability of first passage from } 0 \text { to } 2 k \text { at time } 2 m\}
$$

The probability is given by $[8$, Hitting time theorem (p 79)],

$$
S_{m}(k)=\frac{k}{m}\left(\begin{array}{c}
2 m \\
m+k
\end{array}\right) \frac{(1+\epsilon)}{2}^{m+k} \frac{(1-\epsilon)}{2}
$$

(note that $S_{m}(k)=0$ for $k<m$ ) which may be simplified to

$$
S_{m}(k)=\frac{1}{2^{2 m}}\left(1-\epsilon^{2}\right)^{m}\left(\frac{1+\epsilon}{1-\epsilon}\right)^{k} \frac{k}{m} \frac{(2 m) !}{(m-k) !(m+k) !} .
$$

Using Stirlings formula $n ! \rightarrow n^{n} e^{-n} \sqrt{(2 \pi n)}$ as $n \rightarrow \infty$ we see that

$$
S_{m}(k) \sim \frac{k}{\sqrt{\pi}} e^{m \log \left(1-\epsilon^{2}\right)} m^{-\frac{3}{2}}\left(\frac{1+\epsilon}{1-\epsilon}\right)^{k} .
$$

For our model we assume a threshold level $T=\gamma^{-k}$ implying that the distribution of laminar phases with threshold $T$ is asymptotically

$$
S_{m} \sim \frac{-\log T}{\log \gamma \sqrt{\pi}} e^{m \log \left(1-\epsilon^{2}\right)} m^{-\frac{3}{2}}\left(\frac{1+\epsilon}{1-\epsilon}\right)^{\frac{\log T}{\log \gamma}} .
$$

From this expression we see that the distribution of laminar phases of length $m$ scales as $m^{\frac{-3}{2}}$ for small to moderate values of $m$ and $e^{m \log \left(1-\epsilon^{2}\right)}$ for large values of $m$. For moderate values of threshold level $T$ the distribution of laminar phases reaching a threshold level of $T$ scales linearly with $\log T$ and at high values of $T$ it scales algebraically with $\log T$.

\subsection{Subcritical scenario}

In the subcritical case, $\beta<0$, one obtains a bifurcation from a riddled basin attractor to dynamics where almost all points near the invariant subspace are in the basin of another attractor, namely that at $y=1^{+}$for $-1<\beta<0$ or $y=\infty$ for $\beta<-1$. 
Random walk in the subcritical scenario By considering the map of Section 3 with $\beta<0$ we model the subcritical case and get a random walk model (as derived by [17]) which has the property that points that iterate to $y>1$ are lost to another attractor. We split the phase space into strips $I_{n}$ as in (3.3) and note that $f$ is conjugate to a mapping on the $I_{n}$ (with $I_{k}$ identified for $k<0$ ) defined by

$$
T(x, n)=(g(x), m(x, n))
$$

where

$$
m(x, n)= \begin{cases}n-1 & \text { if } x \leq \alpha \text { and } n \geq 1 \\ n+1 & \text { if } x>\alpha \text { and } n \geq 1 \\ 0 & \text { if } n=0\end{cases}
$$

This gives rise to a transient Markov chain on the non-negative integers where initial states either get trapped in $\left\{I_{k}: k<0\right\}$ or go to $+\infty$, i.e. the invariant subspace.

Measure of the riddled basin before blowout. We investigate the scaling of the measure of the basin of attraction as a function of $\epsilon<0$ where $A$ has a basin of attraction with positive measure. We estimate the measure as a function of the parameter $\epsilon$. The probability of points in $I_{n}$ converging to $A$ is given by the distribution of the asymptotic behaviour of random walks starting at state $n$.

Let $q_{n}$ be the probability that a walk starting at state $n$ is attracted to the state $n=0$; clearly $q_{0}=1, q_{n} \in[0,1]$ and we assume that $q_{n}\left(1-q_{n}\right) \rightarrow 0$ as $n \rightarrow \infty$. We obtain the recurrence

$$
q_{n}=\frac{1+\epsilon}{2} q_{n-1}+\frac{1-\epsilon}{2} q_{n+1}
$$

which we solve to give

$$
q_{n}=\Lambda^{-n}(\epsilon<0), \quad q_{n}=1 \quad(\epsilon \geq 0)
$$

where $\Lambda=\frac{1-\epsilon}{1+\epsilon}$ as before. Thus before the blowout, the probability that a walk starts at state $n$ and is attracted to the invariant subspace is $1-\Lambda^{-n}$. Thus the measure $M(\epsilon)$ of points that are attracted to the invariant subspace is given by

$$
\begin{aligned}
M(\epsilon) & =\sum_{n=1}^{\infty}\left(\gamma^{-n}-\gamma^{-n-1}\right)\left(1-\Lambda^{-n}\right) \\
& =\frac{\Lambda-1}{\Lambda \gamma-1}
\end{aligned}
$$

which to first order in $\epsilon<0$ is

$$
M(\epsilon)=-\frac{2}{\gamma-1} \epsilon+O\left(\epsilon^{2}\right) .
$$

We can use the above result with $\epsilon \geq 0$ to give the following:

Theorem 8 For $\beta<0$ (subcritical case) and $\epsilon \geq 0$ the basin and essential basin of $A$ both have zero measure. 


\section{Discussion}

At the point of blowout bifurcation we see that, at least for the examples discussed, we can distinguish between sub- and supercritical blowout bifurcations by examining the essential basin of attraction at the point of blowout.

- In the supercritical case the set $A$ has essential basin with full measure in a neighbourhood of $A$.

- In the subcritical case the set $A$ has a zero measure essential basin.

In both cases, the basin of $A$ has zero measure and this cannot distinguish criticality. We conjecture that this result holds for generic systems undergoing blowout bifurcation.

Our work was partially motivated by Conjecture 1 in [4]. We show in Theorem 6 that in the supercritical case before blowout, there is a full measure basin (but not necessarily open as conjectured in [4, Conjecture 1(a)]). Moreover, the on-off intermittent attractors that appear in the supercritical case do actually branch from the invariant subspace in a measure-theoretic sense; this confirms [4, Conjecture $1(\mathrm{~b})]$ for a specific family of mappings.

We have investigated scaling of Lyapunov exponents and average distance from the invariant subspace and find in all the cases we have investigated that they scale linearly. We have also found such linear scaling at blowout in a smooth map studied in [3]. In contrast, numerical results in [6] on an $O(2)$-equivariant map found evidence of square-root scaling of the distance from the invariant subspace; reconciling these results is a matter of current research.

This work suggests that there may be many analogies between blowout bifurcation and steady-state bifurcation. In particular, we have made some progress on classifying blowout bifurcations by the codimension of their unfolding (in preparation). This paper has identified some aspects where the analogy to steady bifurcation is broken; for example the possibility of branching to attractors with or without hyperchaos, and the linear scaling of distances from the invariant subspace.

\section{Acknowledgements}

We gratefully acknowledge the support of an EPSRC visiting fellowship to enable MN to visit the University of Surrey during which time this work was undertaken.

\section{References}

[1] J.C. Alexander, I. Kan, J.A. Yorke and Zhiping You. Riddled Basins. Int. Journal of Bifurcations and Chaos, 2:795-813, 1992.

[2] P. Ashwin, J. Buescu and I.N. Stewart. Bubbling of attractors and synchronisation of oscillators. Phys. Lett. A 193:126-139, 1994.

[3] P. Ashwin. Attractors stuck on to invariant subspaces Phys. Lett. A 209:338344, 1995. 
[4] P. Ashwin, J. Buescu and I.N. Stewart. From attractor to chaotic saddle: a tale of transverse instability. Nonlinearity 9:703-737, 1996.

[5] P.J. Aston and M. Dellnitz. Symmetry breaking bifurcation of chaotic attractors, Int. J. Bif. Chaos 5:1643-1676, 1995.

[6] P.J. Aston. Bifurcation and chaos in iterated maps with $O(2)$ symmetry. Int. J. Bif. Chaos 5:701-724, 1995.

[7] A. Cenys and H. Lustfeld. Statistical properties of the noisy on-off intermittency. J. Phys. A Math. Gen. 29:11-20, 1996.

[8] G.R. Grimmett and D.R. Stirzaker. Probability and random processes. Clarendon Press, Oxford, 1992.

[9] P.W. Hammer, N. Platt, S.M. Hammel, J.F. Heagy and B.D. Lee. Experimental observation of on-off intermittency. Phys. Rev. Lett. 73:1095-1098, 1994.

[10] J.F. Heagy, N. Platt and S.M. Hammel. Characterization of on-off intermittency. Phys. Rev. E 49:1140-1150, 1994.

[11] Y.-C. Lai. Symmetry-breaking bifurcation with on-off intermittency in chaotic dynamical systems. Phys. Rev. E 53:R4267-R4270, 1996.

[12] I. Melbourne, M. Dellnitz and M. Golubitsky. The Structure of Symmetric Attractors. Arch. Rational. Mech. Anal. 123:75-98, 1993.

[13] T. Kapitaniak. Transition to hyperchaos in chaotically forced coupled oscillators. Phys Rev E 47:R2975-8, 1993.

[14] J. Milnor. On the concept of attractor. Commun. Math. Phys. 99:177-195, 1985; Comments Commun. Math. Phys. 102:517-519, 1985.

[15] V.I. Oseledec. A multiplicative ergodic theorem: Lyapunov characteristic numbers for dynamical systems. Trans. Mosc. Math. Soc. 19:197-231, 1968.

[16] E. Ott, J.C. Sommerer, J.C. Alexander, I. Kan and J.A. Yorke. Scaling behaviour of chaotic systems with riddled basins. Phys. Rev. Lett. 71:4134-4137, 1993.

[17] E. Ott, J.C. Sommerer, J.C. Alexander, I. Kan and J.A. Yorke. A transition to chaotic attractors with riddled basins. Physica D 76: 384-410, 1994.

[18] E. Ott, J.C. Sommerer. Blowout bifurcations: the occurrence of riddled basins and on-off intermittency. Phys. Lett. A 188:39-47, 1994.

[19] A.S. Pikovsky. Symmetry breaking of chaotic attractors. Z. Phys. B, 55:149-, 1984

[20] A.S. Pikovsky and P. Grassberger. Symmetry breaking of coupled chaotic attractors. J. Phys. A, 24:4587-4597, 1991. 
[21] N. Platt, E.A. Spiegel and C. Tresser. On-off intermittency; a mechanism for bursting. Phys. Rev. Lett. 70:279-282, 1993.

[22] N. Platt, S.M. Hammel and J.F. Heagy. Effects of additive noise on on-off intermittency. Phys. Rev. Lett. 72:3498-3501, 1994.

[23] K. Sigmund. Time averages for unpredictable orbits of deterministic systems. Ann. Operations Research 37:217-228, 1992.

[24] M. da Sousa Vieira, A.J. Lichtenberg and M.A. Lieberman. Synchronisation of regular and chaotic systems. Phys. Rev. A 46:R7359-62, 1992.

[25] T. Yamada and H. Fujisaka. Stability theory of synchronised motion in coupledoscillator systems. Prog. Theor. Phys. 70:1240-8, 1984. 